\title{
The Design of the Orthogonal Box Cavity
}

\author{
A.Moretti, APC \\ $09 / 15 / 10$
}

Introduction:

The muon collider and/or the neutrino factory require large accelerating electric

field gradients immersed in large ( 3 to $6 \mathrm{~T}$ ) solenoidal magnetic fields for

ionization cooling of muon beams. Our original vacuum breakdown study

demonstrated a loss of achievable peak accelerating gradient in solenoidal

magnetic fields by a factor $\mathbf{2}$ or greater. The Muon Collaboration has developed

a theory of a method to suppress high electric field breakdown in vacuum cavities needed for a Muon collider or neutrino factory. It has been shown in our studies and by others that high gradient electric field emitted electrons (dark current) are the primary cause of breakdown. A DC magnetic field orthogonal to the RF electric accelerating field prevents dark current high field emitted electrons from traveling across the accelerating gap and then will prevent breakdown. 
We have decided to test this theory by building a special cavity in the shape of vacuum box. Figure 1 is a simplified view of the cavity design. The design is based on an $805 \mathrm{MHz}$ WR975 waveguide cavity resonating in the TE101 mode.

For the $\mathrm{TE}_{101}$ mode the resonant frequency $\mathrm{f}_{0}$ is given by the relationship

$$
f_{0}=c\left[\left(1 / a^{2}+(m / b)^{2}+(n / d)^{2}\right]^{0.5} / 2 \text { where a and } d\right. \text { are the }
$$

lengths of the base sides and $b$ is the height of the box in MKS units and $c$ is the velocity of light.

\section{Simplified View of Ortogonal Box Cavity}

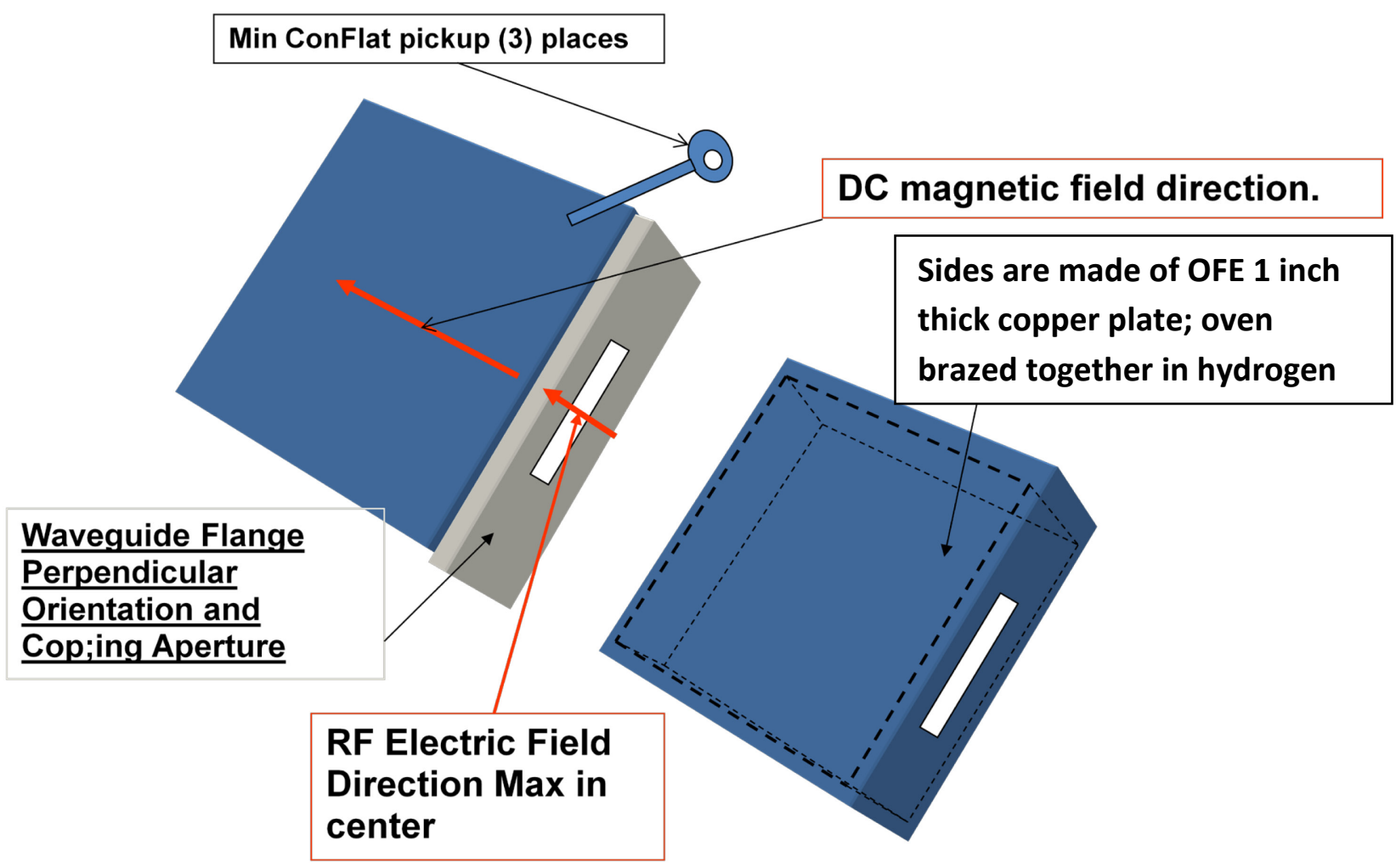

Figure 1, Simplified view of Orthogonal Box Cavity Concept. 
The relationship was first used to set the scale of the simulations for the box

Cavity. The cavity was then optimized by the use of the computer programs

HFSS and Mafia. The program HFSS was used to simulate the cavity with its

Coupling aperture and input drive Waveguide. This allowed us to simulate the

entire problem up to its high power input drive flange, Figure 2.

Orthogonal Box Cavity Coupled to the Waveguide Input

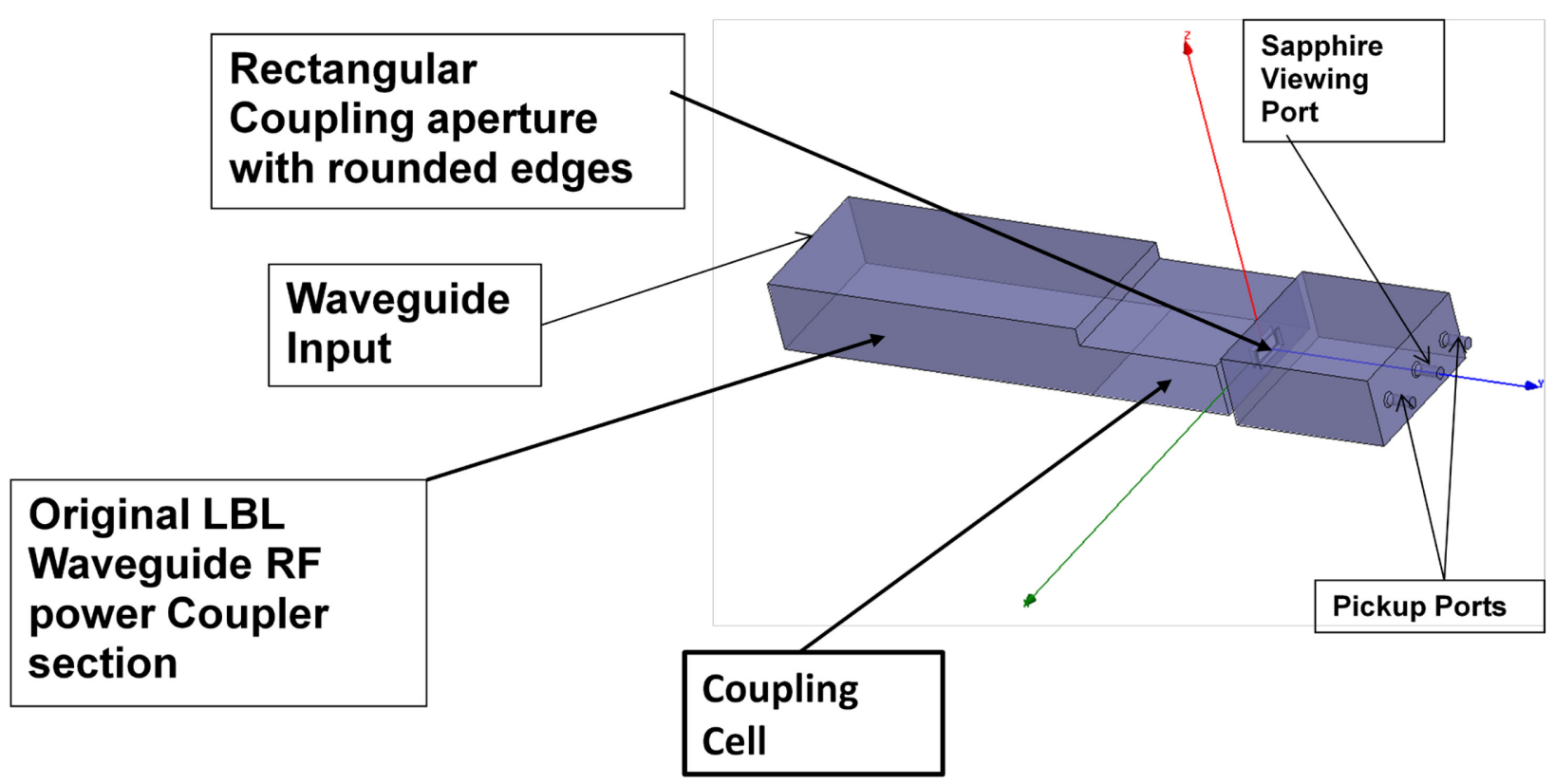

Figure 2, the Entire HFSS Simulation Model 
In addition to fully testing the magnetic insulation theory, the cavity was made rotatable upto 12 Degrees relative to the magnets magnetic field axis.

\section{Design Specifications:}

The orthogonal box cavity is made of 101 OFE copper plates. Interior Parts and the aperture were machined to $32 \mu$ inch finish. Two hydrogen brazing cycles were required to complete the cavity. The HFSS simulation coupling aperture design has been machined into the cavities. The Box Cavity was designed to match the frequency and bandwidth of the "hot" $12 \mathrm{MW}$ peak spare $805 \mathrm{MHz}$ linac klystron. Frequency after final hydrogen brazing $=805 \mathrm{MHz}+/-1 \mathrm{MHz}$

Peak Drive RF power for gradient of $25 \mathrm{MV} / \mathrm{m}=1 \mathrm{MW}$

Tolerance of machined parts $= \pm 0.003 "$

Tolerance after brazing cycles $=+/-0.006 "$

Inside finish $\quad=\mathbf{3 0} \mathrm{u}$ inches

Average power $\quad=$ maximum $5 \mathrm{~kW}$ with half the power

dissipated equally on the Top and bottom plates the remainder almost equally on the 4 sides.

Pick-up ports: Three min-Conflat vacuum feed-thru ports in the side opposite the coupling aperture, 2 for field pick-up loops and 
one for Sapphire vacuum viewing window on a mini Con Flat flange.

Rotation Angle: $90+/-\Delta 12$ Degrees.

Leak check: $<2 x E-10$ ATM cc/sec (He).

\section{HFSS Model and some Simulation Results:}

Figure 3 shows the dimensions of the Box cavity developed by the simulation code HFSS as well as the calculated resonant frequency $\left(F_{0}\right), Q_{0}$, Waveguide match, impedance and $\mathrm{RF}$ power require for gradient of $25 \mathrm{MV} / \mathrm{m}$. The design frequency for the cavity was free parameter and was chosen as $805.2 \mathrm{MHz}$ at the maximum output power frequency of the $12 \mathrm{MW}$ drive klystron. 
HFSS Cavity dimensions and Parameters of Box cavity

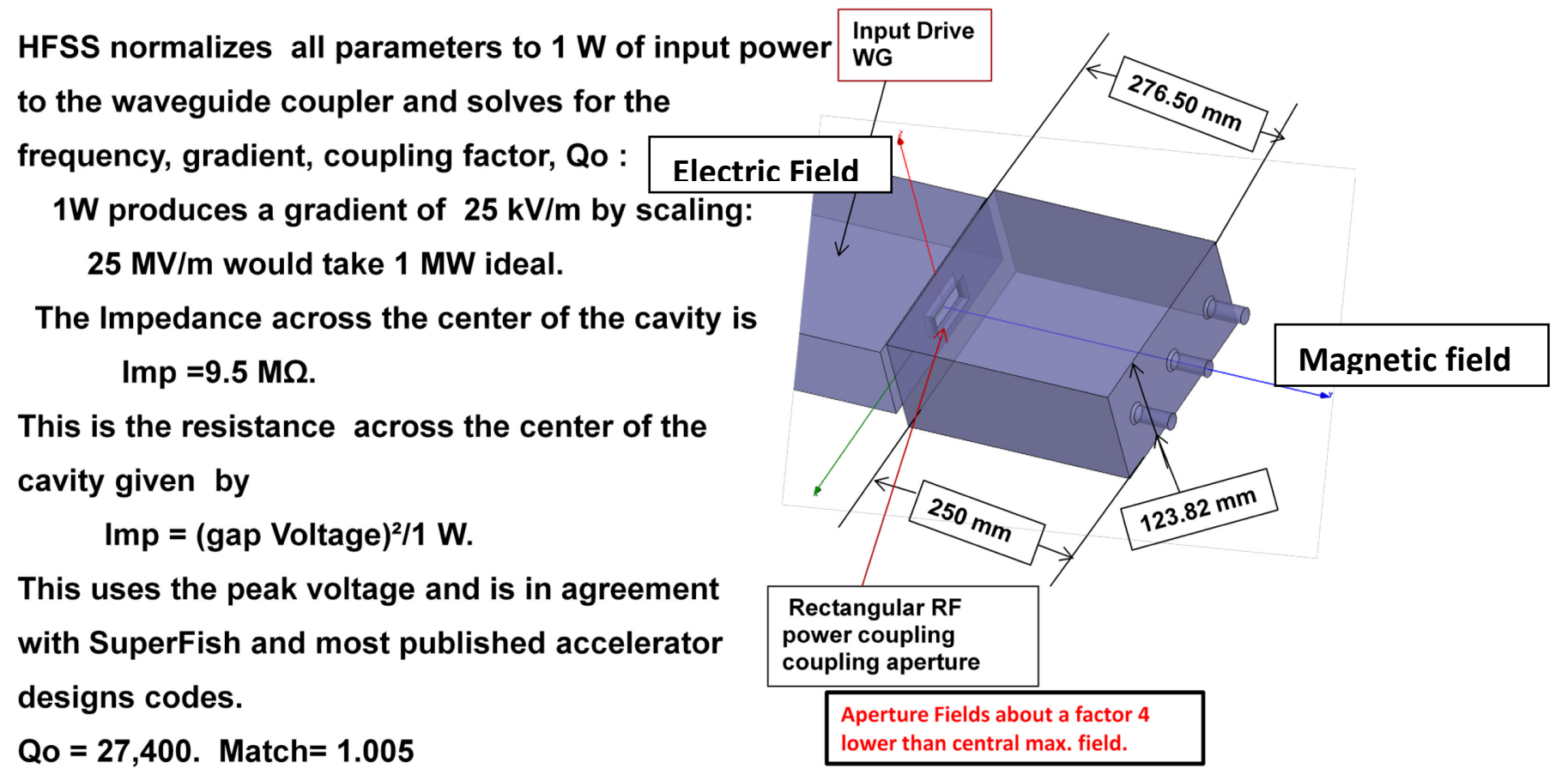

Figure 3 HFSS Model and some Simulation Results 


\section{Note 1:}

Ratio Eps/ Epa $=3.5$

for orthogonal case.

\section{Note 2:}

Ratio Eps/ Epa= 2

for parallel case.

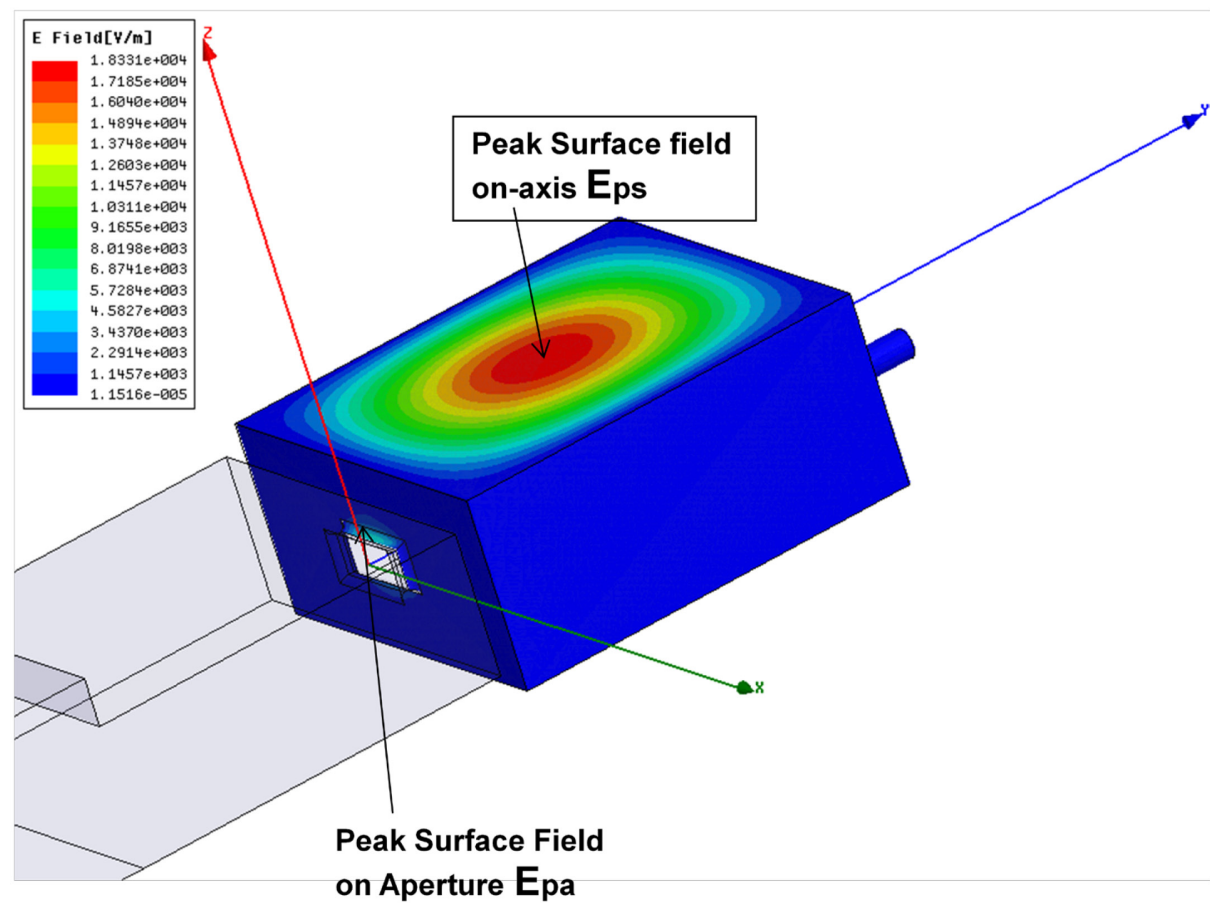

\section{Picture of Assembled Cavity in Test Lab and Test results:}

After the final brazing at the factory in California, the cavity was returned to

Fermilab for testing in the $\mathrm{A} 0$ superconducting test lab. The cavity was first

Cleaned using superconducting techniques and_successfully vacuum checked.

The cavity was then connected to a network analyzer for frequency ( $\left.F_{0}\right), Q_{0}$, and

Waveguide input power match. 


\section{Box Cavity in A0 Test Lab after successful Vacuum Leak Check}

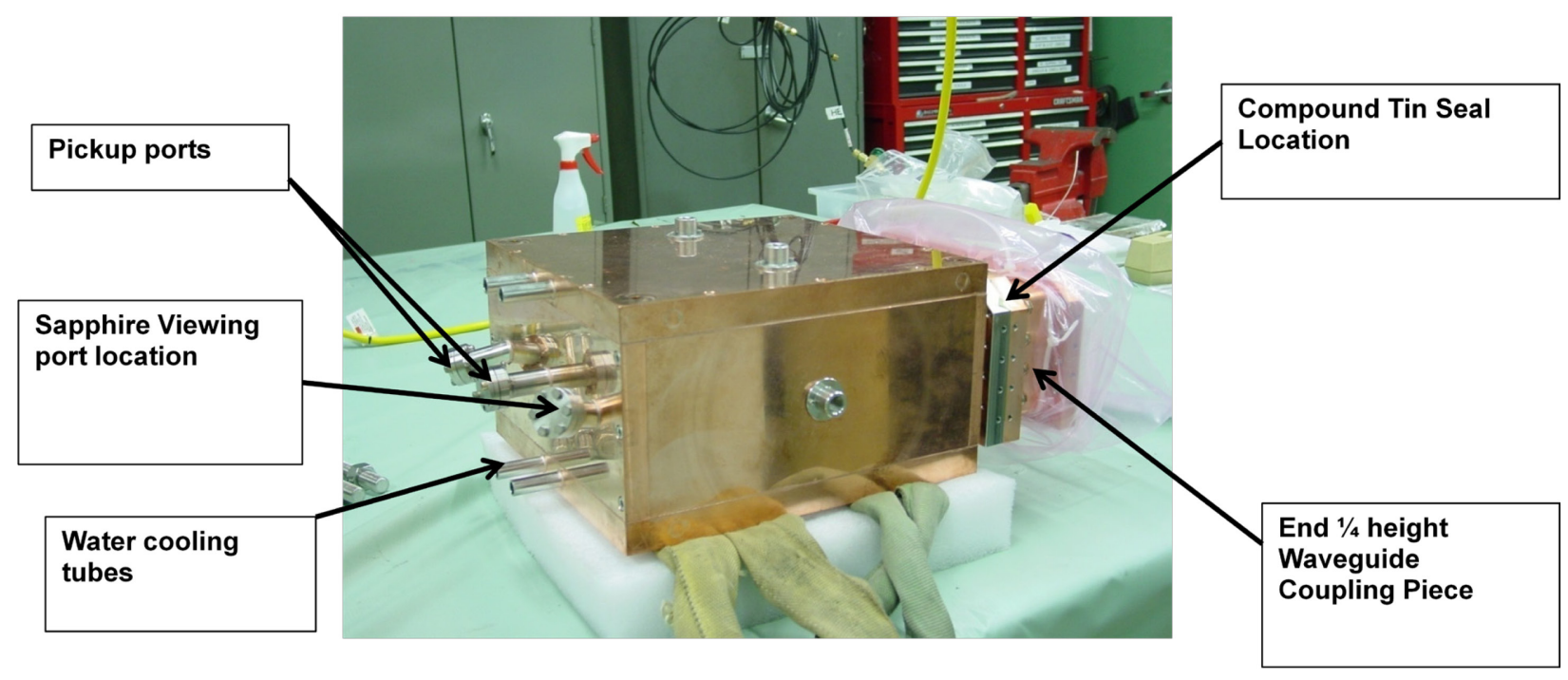

Figure 4, Cavity Test Bench in AO Lab. 


\section{Cavity attached to Tapered waveguide coupler and WG-typeN Adapter.}

Network analyzer measurements:

- Fo = 805.33 MHz test lab; Simulation $\mathrm{Fo}=806.2 \mathrm{MHz}$

- $Q 0=27,400$;

Simulation Qo $=27,400$

- $\beta=0.96$ coupling factor; Simulation $\beta=1.06$.

-These values are preliminary and may change when attached to the LBL stepped WG coupler and mounted in the magnet. Even with a coupling factor of $\beta=$ $0.96,99.95 \%$ of the transmitted RF power go into the cavity.

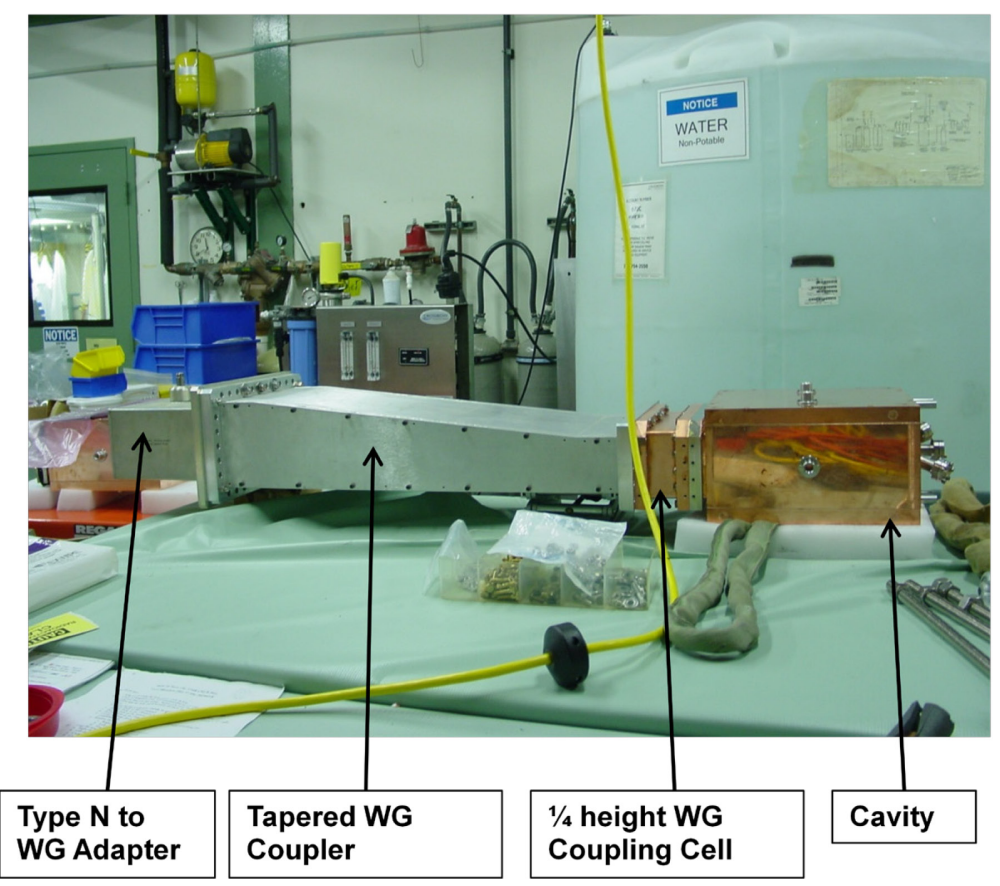

Figure 5, Test Setup before Attachment to Network Analyzer 